\title{
A Cross-Platform Consumer Behavior Analysis of Large-Scale Mobile Shopping Data
}

\author{
Hong Huang* \\ Huazhong University of Science and \\ Technology \\ Wuhan, China \\ honghuang@hust.edu.cn \\ Zhou Zhuang \\ Fudan University \\ Shanghai, China \\ 14307130071@fudan.edu.cn \\ Xinggang Wang \\ China TelecomCo., Ltd. \\ Beijing, China \\ wangxingg@chinatelecom.cn
}

\author{
Bo Zhao \\ Gesellschaft Fur Wissenschaftliche \\ Datenverarbeitung mbH Goettingen \\ (GWDG) \\ Goettingen, Germany \\ bo.zhao@gwdg.de \\ Zhenxuan Wang \\ University of Goettingen \\ Göttingen, Germany \\ zxwang1497@gmail.com \\ Hai Jin \\ Huazhong University of Science and \\ Technology \\ Wuhan, China \\ hjin@hust.edu.cn
}

\author{
Hao Zhao \\ Fudan University \\ Shanghai, China \\ haozhao14@fudan.edu.cn
}

\begin{abstract}
The proliferation of mobile devices especially smart phones brings remarkable opportunities for both industry and academia. In particular, the massive data generated from users' usage logs provide the possibilities for stakeholders to know better about consumer behaviors with the aid of data mining. In this paper, we examine the consumer behaviors across multiple platforms based on a largescale mobile Internet dataset from a major telecom operator, which covers 9.8 million users from two regions among which 1.4 million users have visited e-commerce platforms within one week of our study. We make several interesting observations and examine users' cultural differences from different regions. Our analysis shows among the multiple e-commerce platforms available, most mobile users are loyal to their favorable sites; people (60\%) tend to make quick decisions to buy something online, which usually takes less than half an hour. Furthermore, we find that people in residential areas are much easier to perform purchases than in business districts and purchases take place during non-work time. Meanwhile, people with medium socioeconomic status like browsing and purchasing on e-commerce platforms, while people with high and low socioeconomic status are much easier to conduct purchases online. We also show the predictability of cross-platform shopping
\end{abstract}

*1. H. Huang and H. Jin are with Service Computing Technology and System Lab / Big Data Technology and System Lab in Huazhong University of Science and Technology. 2. H. Huang and X. Fu are the corresponding authors.

3. Part of this work was done while H. Huang was with the University of Goettingen, Germany.

This paper is published under the Creative Commons Attribution 4.0 International (CC BY 4.0) license. Authors reserve their rights to disseminate the work on their personal and corporate Web sites with the appropriate attribution.

WWW 2018, April 23-27, 2018, Lyon, France

() 2018 IW3C2 (International World Wide Web Conference Committee), published under Creative Commons CC BY 4.0 License.

ACM ISBN 978-1-4503-5639-8/18/04.

https://doi.org/10.1145/3178876.3186169 behaviors with extensive experiments on the basis of our observed data. Our discoveries could be a good guide for e-commerce future strategy making.

\section{CCS CONCEPTS}

- Applied computing $\rightarrow$ Online shopping; Law, social and behavioral sciences; • Information systems $\rightarrow$ Data mining; • Human-centered computing $\rightarrow$ Empirical studies in ubiquitous and mobile computing; $\bullet$ Computing methodologies $\rightarrow$ Machine learning;

\section{KEYWORDS}

mobile usage, e-commerce, consumer behavior, data mining, predictability

\section{ACM Reference Format:}

Hong Huang, Bo Zhao, Hao Zhao, Zhou Zhuang, Zhenxuan Wang, Xiaoming Yao, Xinggang Wang, Hai Jin, and Xiaoming Fu. 2018. A Cross-Platform Consumer Behavior Analysis of Large-Scale Mobile Shopping Data. In WWW 2018: The 2018 Web Conference, April 23-27, 2018, Lyon, France. ACM, New York, NY, USA, 10 pages. https://doi.org/10.1145/3178876.3186169

\section{INTRODUCTION}

With the development of smart phones and mobile applications, people are spending more and more time on mobile devices. According to a recent survey, nearly 75 percent of US adults will use a smartphone in 2017. On average people spend 3 hours and 15 minutes per day on a mobile device ${ }^{1}$. In November 2016, the mobile Internet usage even surpassed desktop usage for the first time ${ }^{2}$. The proliferation of mobile usage has already shaped our lives (e.g., conquered our wallets) and dramatically changed the business models for numerous enterprises. A study shows that the majority of online

\footnotetext{
${ }^{1}$ http://www.geomarketing.com/us-mobile-usage-in-2017-stats-you-need-to-know ${ }^{2}$ http://bgr.com/2016/11/02/internet-usage-desktop-vs-mobile/
} 
shopping sales in the UK are now conducted through smartphones and tablets, instead of traditional computers or laptops ${ }^{3}$.

The popularity of mobile devices and the massive data generated from mobile usage offers the research community unprecedented opportunities to study mobile user behavior patterns, which were previously difficult to explore due to a lack of sufficient data. A better understanding of user behavior and underlying usage patterns can allow a mobile service provider to define effective marketing strategies for attracting more users and maintaining current users, eventually increasing its profit. An example is the story of beer and diapers ${ }^{4}$ which suggests an innovative marketing strategy when analyzing supermarket consumer behavior data. For individual users, a better understanding of their own temporal behavior patterns can help them better plan their own household budgets and make better use of the provider's marketing strategies.

With the emergence and ever increasing number of online shopping platforms, users have more possibilities to do their shopping online. They may move across different online platforms to search for their ideal products with considering complex factors, such as nice price, good service or sales. However, due to the limitations of lack of data, previous work has mainly focused on user behavior analysis of single e-commerce platforms [31]. It is still unclear whether people will move across different shopping platforms and even why and how the users jump from one platform to the next.

In addition, users' profiles such as their culture, social and ethical and as well as the functional regions they belong to would also influence their behaviors [26]. Researchers pay more attention to users profiling $[2,7,11,13,31]$ and apply them in many areas, such as personalization and recommender systems [1, 15, 19, 25]. Whether and how users' profile (e.g., app usage behaviors), their functional zones and socioeconomic status would influence their shopping decisions will also provide useful insights.

Thanks to the e-commerce big data associated with smart phones, it is now possible to correlate a single user's shopping behavior across multiple platforms and with large-scale mobile usage logs, we are able to access all the platforms that users have visited although it also brings us challenges during accessing and processing the data. For instance, the size of compressed mobile Internet data usage records including active online shopping activities for 10 million mobile phone users during one week could easily exceed $40 \mathrm{~TB}$, which were used in the scenario of this paper.

In this paper, employing a large mobile communication data from a major telecom provider in two populous regions in China over a period of one week as the basis in our study, we systematically investigate the problem of cross-platform and cross-region consumer shopping behaviors. We first try to answer the following 6 questions:

(1) how spatiotemporal factors influence users' shopping behaviors;

(2) how users' shopping behaviors vary in different functional zones;

(3) whether users' profile (e.g., app usage behaviors) and socioeconomic status would influence their shopping decisions;

\footnotetext{
${ }^{3}$ http://www.telegraph.co.uk/news/shopping-and-consumer-news/12172230/ Are-mobiles-changing-how-we-shop.html

${ }^{4}$ https://www.theregister.co.uk/2006/08/15/beer_diapers/
}

(4) how do people make their shopping decisions;

(5) whether users exhibit signs of loyalty to certain shopping platforms;

(6) whether users' cross-platform shopping behaviors are predictable.

We made several interesting observations. For example, among the multiple e-commerce platforms available, most mobile users are loyal to their favorable sites; people $(60 \%)$ tend to make quick decisions to buy something online, which usually takes less than half an hour. People in residential areas are much easier to perform purchases and they prefer to purchasing during non-work time. Furthermore, people with medium socioeconomic status like browsing and purchasing on e-commerce platforms, while people with high and low socioeconomic status are much easier to conduct purchases online.

Based on the observations, we further examine the predictability of cross-platform shopping behaviors. We build a framework with four types of features: temporal feature, loyalty feature, profiling feature and demographic feature. The prediction results show that consumers' cross-platform shopping behaviors are predictable and our prediction performance is as high as $94 \%$ in terms of both F1 and accuracy.

The rest of this paper is organized as follows. Section 2 introduces the dataset used in our study. Section 3 examines how spatiotemporal factors influence users' shopping behaviors. Section 4 determines whether users' shopping behaviors vary in different functional zones. Section 5 shows whether users' profile would influence their shopping decisions. Section 6 explores users' shopping decision making behaviors. Section 7 studies users' loyalty across different shopping platforms. Section 8 presents our prediction framework and prediction results and Section 9 gives related works. Section 10 concludes.

\section{DATASET}

Data Collection. The dataset is drawn from a log of anonymized browsing records of mobile usage in cellular environments provided by one of the three major mobile telecom operators in China. It contains the mobile usage data for over $9,700,000$ users from two populous regions over a period of roughly one week each: one is Shanghai, the most populous metropolitan in the world (and also the commercial and financial center of mainland China), between April 20 and April 26, 2016 and the other is Shandong province, the second most populous province of China, with only $45 \%$ of per capita disposable income of Shanghai ${ }^{5}$, between August 6 and August 14, 2016. Each of these records contains the anonymized ID of the mobile device and the start time for each action, as well as browsing records. Part of these records contain geo-location information in the forms of longitude and latitude where the action was performed.

Data Pre-processing. The collected data is heterogeneous and noisy. In order to study consumer behavior using these vast mobile browsing records, we need to begin by cleaning the data.

We analyzed the 5 most popular Chinese B2C e-commerce platforms, which are Taobao (taobao.com), JD (jd.com), Suning (suning.com), Dangdang (dangdang.com) and Vip (vip.com). We focused

\footnotetext{
${ }^{5}$ http://www.yicai.com/news/5229761.html
} 

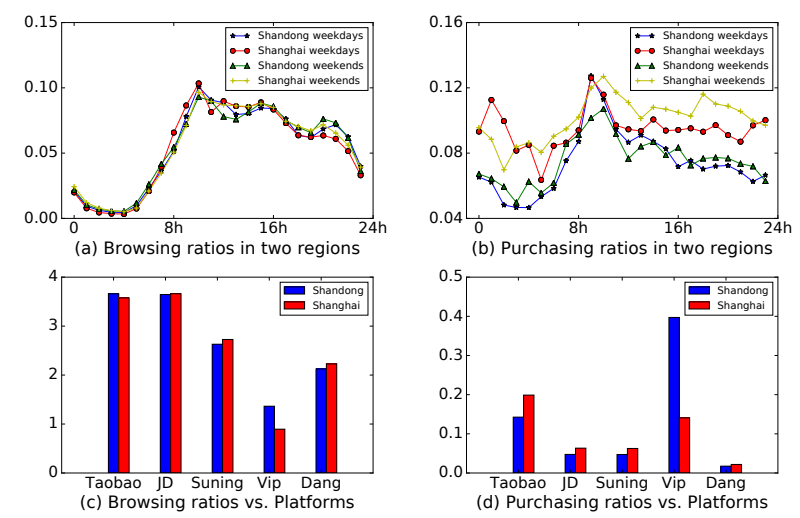

Figure 1: How spatiotemporal factors influence users' shopping behaviors? (a) and (b) show the browsing and purchasing ratios in two regions respectively, with the $\mathrm{X}$-axis being the hour in a day, and Y-axis being browsing or purchasing ratios where the ratio means the percentage of purchases within one hour to the total number browses within a day. (c) and (d) show the browsing and purchasing ratios on diverse platforms, with $\mathrm{X}$-axis unit being diverse platforms and $Y$-axis being browsing or purchasing ratios. Notify that in order to make the figure more readable, in (c) we make the Y-axis as $\log 10$ (actual browsing ratios* 10000).

on all users who browsed or purchased on these platforms, and extracted all browsing and purchasing records. Due to the multiple interaction rounds of web service requests and response queries on various platforms, a single browsing or purchasing action needed to be identified from many redundant interaction records. To make it simple, we only counted each page visit once. After eliminating redundant records, we obtained 386,379 unique browsing and purchasing records. The detailed data statistics is shown in Table 1.

\section{HOW SPATIOTEMPORAL FACTORS INFLUENCE USERS' SHOPPING BEHAVIORS?}

In this part, we will examine how spatiotemporal factors (e.g., time, regions and platforms) influence users' shopping behaviors, i.e., product browsing or purchasing.

Influence of Time. People usually have different time schedules on weekdays and weekends in different regions. Fig. 1(a) and (b) show users' browsing and purchasing behaviors separately in Shanghai and Shandong during different time periods. From Fig. 1(a), we can see that people tend to have the similar browsing behaviors on both weekdays and weekends. For example, people in both Shandong and Shanghai are willing to browse shopping websites during the morning coffee break (i.e., around 10:00).

In addition, people prefer to browse shopping pages during working hours (8:00 - 17:00). In terms of purchasing behavior, people are more willing to pay for their orders around $11 \mathrm{am}$, which is right after the time most people spend browsing. What's more, people prefer to place their orders on weekends versus weekdays according to Fig. 1(b).
Influence of Platforms. We focus primarily on the 5 most popular Chinese B2C e-commerce platforms. Here we will examine users' shopping behaviors over each platform separately. Fig. 1 (c) and (d) show users' browsing and purchasing behaviors on each platform.

From Fig. 1(c), we can see that Taobao and JD are the most popular platforms, which are the two largest and most comprehensive online shopping platforms in China, making up $74.0 \%$ of the browsing records and $93.9 \%$ of the purchases from our dataset. Interestingly, we find that people are more willing to purchase on Taobao and Vip, as shown in Fig. 1(d).

Influence of Regions. From Fig. 1(a), we can see that people in different regions tend to have similar browsing behaviors. However, they react quite differently when making purchases. From Fig. 1(b), we can see that users from Shanghai are more likely to carry out online shopping purchases than people in Shandong. This might be due to that people from less developed regions are more concerned about spending their hard earned money. Furthermore, Shanghai consumers tend to carry out purchases late at night or in the early morning hours, versus consumers in Shandong, which might be a reflection of Shanghai's socio-economic situation, as the business and financial center of China.

\section{HOW USERS' SHOPPING BEHAVIORS VARY IN FUNCTIONAL ZONES?}

The modern civilization and urbanization fosters functional zones in a city [30] and people behave differently in various zones. In this section, we examine whether users' shopping behaviors vary in functional zones.

In this paper, we divide a city into four types of functional zones: business districts, residential areas, leisure areas and others. Since we only have users' geo-locations, we determine these functional zones according to the Point of Interest (POI) associated with these locations ${ }^{6}$. POI labels associated with each functional zones are shown in Table 2.

We now check users' shopping behaviors in each type of functional zones. From Figure 2, we can see that people in business districts perform the highest number of browsing and purchasing activities. However, people in residential areas are mostly like to make purchase decisions. This is partly because in business districts, people are more likely to visit e-commerce platforms in cellular environments, thus having more browsing and purchasing records. Yet, it is easier for people to make purchase decisions in residential areas.

Users'Shopping Behavior in Work Time vs. Off Work Time. In addition, we check users' shopping behaviors in each functional zone in different time slots. We divide a day into work time and off work according to their working state (i.e., $9 \mathrm{am}-6 \mathrm{pm}$ is for work time and the others for off work time.). From Figure 3, we can see that people in business districts tend to have more browsing and purchasing activities in work time while people in residential areas tend to have more purchases in off work time, which is quite coincident with our intuition.

Users' Shopping Behaviors vs. Socioeconomic Status. In this part, we examine whether users' socioeconomic status will influence their shopping behaviors. "Socioeconomic status is the social

${ }^{6}$ The POI dataset is public under the link: http://pan.baidu.com/s/1pKCL6YZ. 
Table 1: Dataset Statistics

\begin{tabular}{l|r|r}
\hline Item & Shandong & Shanghai \\
\hline Time period & Aug 6th - Aug 14th, 2016 & Apr 20th - Apr 26th, 2016 \\
\#Mobile User & $5,461,244$ & $4,309,914$ \\
\#Average user per day & $2,827,771$ & $2,914,294$ \\
\#Online shopper & 301,426 & 233,537 \\
\#Average shopper per day & 45,481 & 47,579 \\
\#Purchaser & 33,189 & 35,041 \\
\#Average purchaser per day & 3,970 & 5,454 \\
\#Browsing records & 156,019 & 135,154 \\
\#Average browsing records per day & 17,335 & 19,308 \\
\#Purchase & 40,753 & 54,453 \\
\#Average purchase per day & 4,528 & 7,779 \\
\hline
\end{tabular}

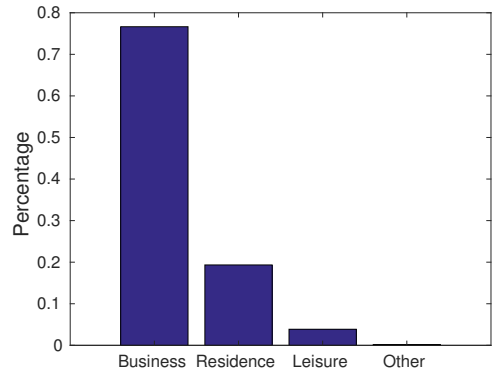

(a) Browsing

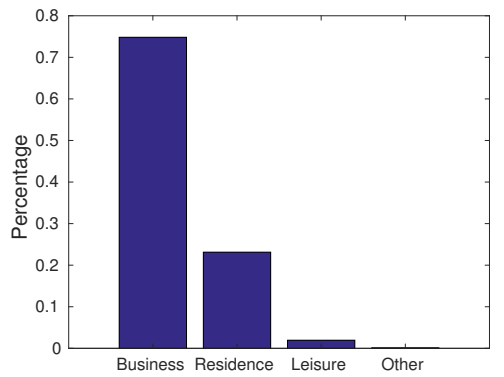

(b) Purchasing

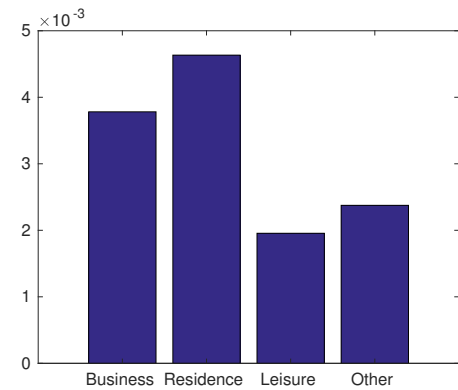

(c) Purchasing/Browsing

Figure 2: Users' shopping behaviors in 4 functional zones. (a) browsing behavior; (b) purchasing behavior; (c) the ratio of purchase.

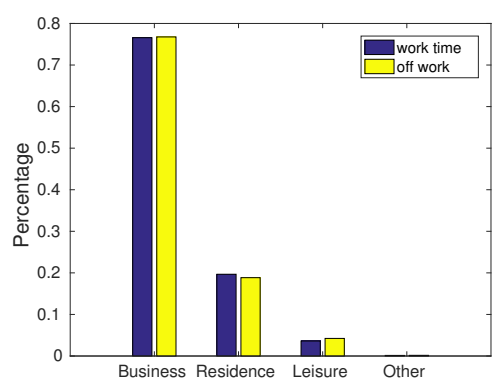

(a) Browsing

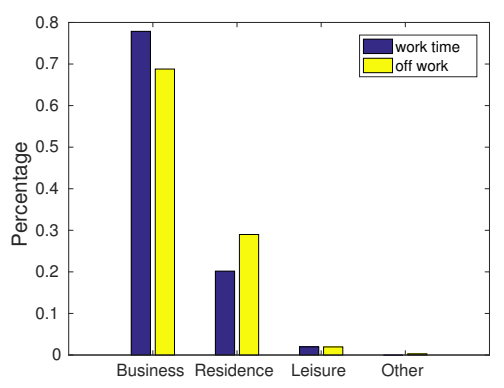

(b) Purchasing

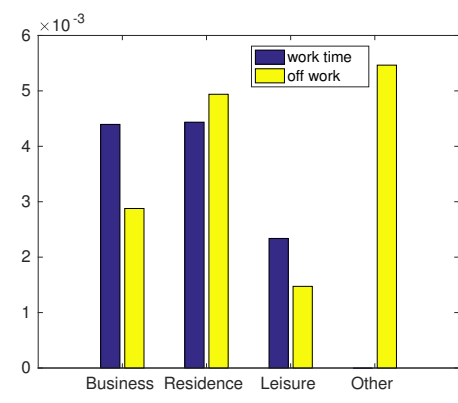

(c) Purchasing/Browsing

Figure 3: Users' shopping behaviors in work time vs. off work in 4 functional zones. (a) browsing behavior; (b) purchasing behavior; (c) the ratio of purchase.

Table 2: Identification of Functional Zones Based on POI

\begin{tabular}{c|c}
\hline Zones & POI labels \\
\hline Business & government; education; hospital; company; etc. \\
Residence & town; village; villa; realty; etc. \\
Leisure & hotel; sport; scenery; restaurant; shopping; etc. \\
Other & others. \\
\hline
\end{tabular}

standing or class of an individual or group. It is often measured as a combination of education, income and occupation." [28]. However, due to privacy reasons, it is difficult to map the education, income and occupation information of an individual to the online shopping data. Hence, we use the housing price ${ }^{7}$ associated with a user's residential address (geo-location) to approximate the user's socioeconomic status, and divide people's socioeconomic status

\footnotetext{
${ }^{7}$ The house price is crawled from Lianjia (lianjia.com), one of the most famous real estate agency platforms in China.
} 

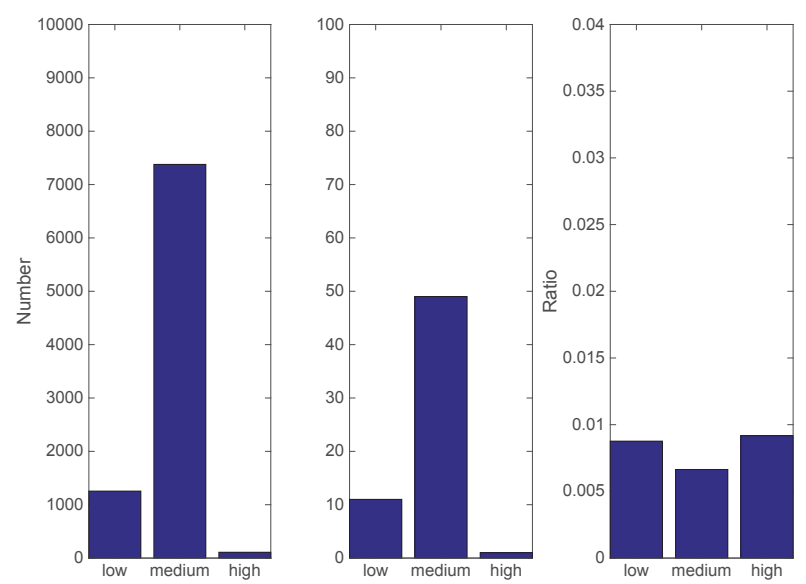

Figure 4: Users' shopping behaviors vs. their socioeconomic status. We divide people's socioeconomic status into three categories: high, medium and low, which is shown in $x^{-}$ axis. Left: browsing behavior; Middle: purchasing behavior; Right: the ratio of purchase.

into three rough categories: high, medium and low ${ }^{8}$. Figure 4 shows the results. From the figure, we can see that people with medium socioeconomic status like browsing and purchasing on e-commerce platforms, while people with high and low socioeconomic status are much easier to conduct purchases online.

\section{DO USERS' APP USAGE BEHAVIORS MATTER?}

Users have their own shopping behaviors and preference towards the usage of apps on smart phones. Usually, a user's app usage behaviors could well profile and characterize the user. In this section, we will examine whether users' apps usage behavior will influence their shopping decisions. We will first classify all users' app usage behaviors and then check the correlation between app usage behaviors and shopping behaviors.

What Apps Do People Always Use? According to a report by Nielsen ${ }^{9}$, users spent most of their time (84\%) on smart phones on just 5 non-native apps. The five apps will vary from person to person and show personality of this user. For some user, their top five could include social media or gaming, while others may spend more time in instant messaging. To this end, we analyze one user's app usage behavior using his five most frequent used ones.

However, due to the limitation in data availability, we cannot directly know which apps people are using with users' mobile visiting records, based on the available information concerning the urls of web visiting with domain names. Thus, we need first parse these records with domains names in order to understand the apps people are using.

In total, we obtain 8,898 unique domain names for various apps from 12,385 mobile users. We then cluster these domain names into

\footnotetext{
${ }^{8}$ Price lower than $40,000 \mathrm{CNY}$ per $m^{2}$ as low, higher than 70,000 as high and others as medium.

${ }^{9}$ https://techcrunch.com/2015/06/22/consumers-spend-85-of-time-on-smartphonesin-apps-but-only-5-apps-see-heavy-use/
}

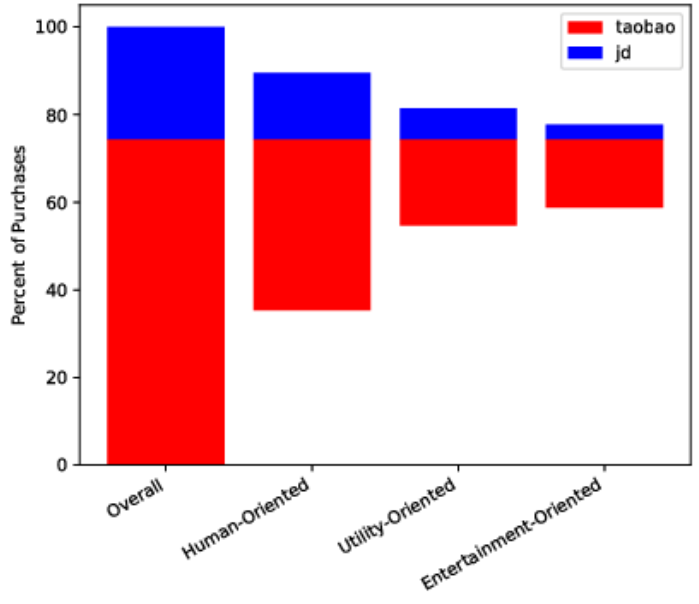

Figure 5: Correlations between users' apps usage behaviors and their shopping decisions. $\mathrm{X}$-axis: overall cases and three categories. Y-axis: Percent of purchases.

several clusters using DBSCAN method [8] under the Levenshtein Distances [17].

The 8,898 domain names are clustered into 393 clusters. We then manually labeled these clusters with the reference to app names in Xiaomi app store, one of the largest mobile app store in China. In this way, we get the apps people use from their mobile internet usage records.

Users' App Usage Behaviors vs. Shopping Behaviors. Based on the apps' functionality, we classify all users' apps usage into three categories: Human-oriented apps, Utility-oriented apps, and Entertainmentoriented apps. Human-oriented apps represents apps that serves people's basic needs in daily lives such as "Shopping", "Health" and "Lifestyle", etc. Utility-oriented apps are for utility perspective such as "Travel" and "Photography", while Entertainment-oriented apps included apps for leisure such as "Games". We then check whether people's app usage behaviors will influence their shopping behaviors.

Since JD and Taobao are the two major online shopping platforms in China, we only consider the correlation between users' apps usage behaviors and their shopping decisions on these two platforms. Figure 5 shows the results. From this figure, we can see that users who prefer human-oriented apps are more likely to buy goods online as they pay more attention to "Shopping".

\section{HOW LONG IT TAKES ONE USER TO MAKE HIS DECISION TO PURCHASE?}

In this part, we would like to focus on the question regarding "how long it takes one user to buy a product?". For simplicity's sake, we assume that each purchase is independent. That is, a user will start a new purchase only after he ends the last one. Based on our observations, more than $96 \%$ of purchases take less than 4 days to carry out, so that we have focused on a 4-day time period before each purchase. We divide the time period into several time frames, 
Table 3: Types of Users
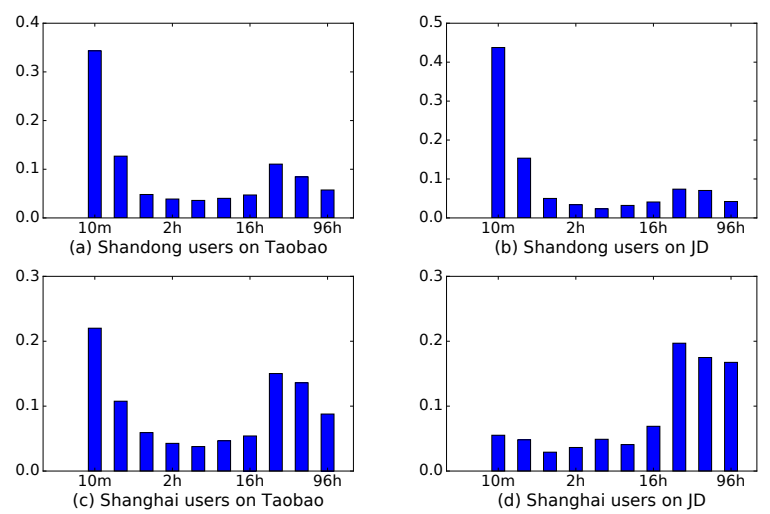

Figure 6: How much time it takes you for one successful purchase? X-axis: time frame $\{0-10 \mathrm{~m}, 10 \mathrm{~m}-30 \mathrm{~m}, 30 \mathrm{~m}-1 \mathrm{~h}, 1 \mathrm{~h}$ 2h, 2h-4h, 4h-8h, 8h-16h, 16h-32h, 32h-64h, 64h-96h\}; Y-axis: browsing ratios.

namely $\{0-10 \mathrm{~m}, 10 \mathrm{~m}-30 \mathrm{~m}, 30 \mathrm{~m}-1 \mathrm{~h}, 1 \mathrm{~h}-2 \mathrm{~h}, 2 \mathrm{~h}-4 \mathrm{~h}, 4 \mathrm{~h}-8 \mathrm{~h}, 8 \mathrm{~h}-16 \mathrm{~h}, 16 \mathrm{~h}-$ $32 \mathrm{~h}, 32 \mathrm{~h}-64 \mathrm{~h}, 64 \mathrm{~h}-96 \mathrm{~h}$. We then observed users' browsing records in each time frame. For this study, we only consider the largest two shopping platforms - Taobao and JD.

Time for Decision Making. Fig. 6 shows users' page visiting counts in each time frame. From the figure we can see that when people shop on Taobao, they visit the shopping pages frequently, which is shortly before each purchase. If a user only browses product pages shortly before his final purchase without any previous visits, we can say that he is quick purchaser as he usually spends little time on thinking about the purchase.

However, when shopping on JD, Shandong users tend to make quick decisions, whereas Shanghai users tend to spend more time thinking about their shopping purchases.

Group of People that Have Similar Shopping Decision Making Behaviors. We know that some users are quick to make purchases while others need more time to think about their purchases. For sellers, this information can assist them in further developing more personalized marketing strategies.

According to our observations, the consumer is apt to visit a webpage more frequently, the closer he is to making a final purchase. In order to explore this feature more closely, we divide the 4-day time period into the following time frames in an exponentially increasing manner $\{0-10 \mathrm{~m}, 10 \mathrm{~m}-20 \mathrm{~m}, 20 \mathrm{~m}-30 \mathrm{~m}, 30 \mathrm{~m}-40 \mathrm{~m}, 40 \mathrm{~m}-$ 50m, 50m-1h, 1h-2h, 2h-4h, 4h-6h, 6h,12h, 12h-18h, 18h-24h, 24h36h, 36h-48h, 48h-60h, 60h-72h, 72h-84h, 84h-96h\}. Drawing on these browsing behaviors in each time frame, we can cluster users into several groups. We use K-Spectral Centroid (K-SC) clustering algorithm [29] and set the number of cluster as 4. Fig. 7 illustrates these clusters.

As illustrated in the figure, the vast majority of users tend to visit selected pages for 30 minutes before a final purchase. This makes sense since we often check the status of a product before a final order is placed.

The four types of users are as follows:

\begin{tabular}{c|c|l}
\hline Type ID & Type Name & Percentage \\
\hline I & Quick purchaser & $61.2 \%$ \\
II & Hesitant user & $21.93 \%$ \\
III & Short-term decision-maker & $9.53 \%$ \\
IV & Long-term decision-maker & $7.24 \%$ \\
\hline
\end{tabular}
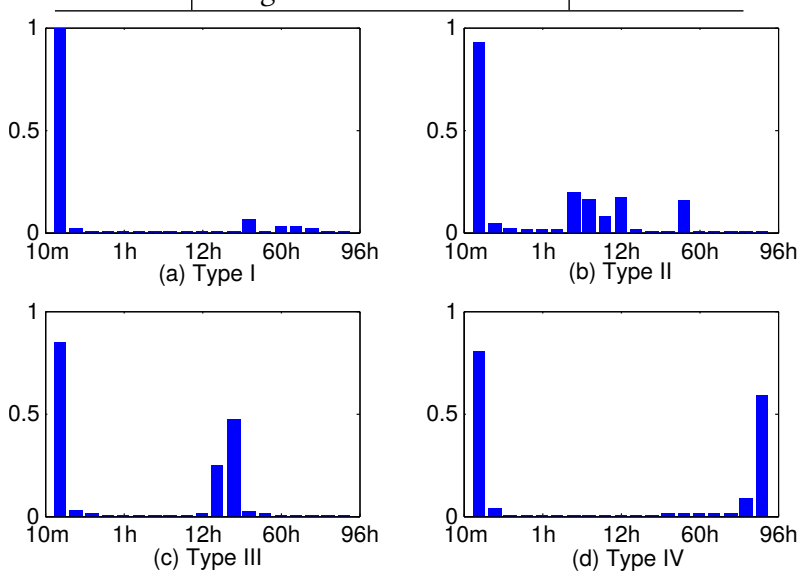

Figure 7: Illustration of four types of users based on their browsing behaviors. $X$-axis: time frame $\{0-10 \mathrm{~m}, 10 \mathrm{~m}-20 \mathrm{~m}$, 20m-30m, 30m-40m, 40m-50m, 50m-1h, 1h-2h, 2h-4h, 4h-6h, 6h,12h, 12h-18h, 18h-24h, 24h-36h, 36h-48h, 48h-60h, 60h72h, 72h-84h, 84h-96h\}; Y-axis: browsing ratios. (a) Quick purchaser; (b) Hesitant user; (c) Short-term decision-maker; (d) Long-term decision-maker.

Type I - users will browse the pages for less than 30 minutes before a purchase. We refer to these users as quick purchasers. Over $60 \%$ of users are such "quick purchasers".

Type II - users will sporadically keep returning to shopping pages, as they have a hard time during making decisions. We refer to these users as "hesitant users".

Type III - users generally browse the pages for 12 hours before their purchase, so that we might assume that they spend about half a day to make a decision. We refer to these users as "short-term decision-makers".

Type IV - users who need nearly 4 days to make a decision, thus we refer to them as "long-term decision-makers".

Among all 4 types, quick decision-makers comprise the largest group, which is around $60 \%$ of users, followed by hesitant users, which comprises about $20 \%$ of consumers. The remaining $20 \%$ of users belong to either of the other two user types. These four clusters of users and their distribution is summarized in Table 3.

\section{ARE CONSUMERS LOYAL?}

Many users choose to visit their preferred shopping platforms and do not want to try others, while other users will move across different platforms to search for the best deals. If users continually visit their preferred shopping platforms, we refer to these users as loyal users. In this section, we will answer the question "Are users loyal to certain shopping platforms and to which extent?". In other words, to which extent do users use the same shopping platform 
Table 4: The top 12 behavior patterns

\begin{tabular}{c|c}
\hline & Behaviors patterns \\
\hline 1 & JD_browsing \\
\hline 2 & JD_browsing, JD_purchasing \\
\hline 3 & Taobao_browsing, Taobao_purchasing \\
\hline 4 & Taobao_browsing \\
\hline 5 & Suning_browsing, Suning_purchasing \\
\hline 6 & Vip_browsing \\
\hline 7 & Suning_browsing \\
\hline 9 & Taobao_browsing, JD_browsing \\
\hline 10 & Vip_browsing, JD_browsing \\
\hline 11 & Vip_browsing, Vip_purchasing \\
\hline 12 & Suning_browsing, JD_browsing \\
\hline
\end{tabular}

and to which extent do they move across different platforms in search of the best deal?

What Do People Usually Do During One Purchase? According to users' shopping behaviors, it is possible to identify multiple behavior patterns that take place during purchases. Unlike traditional frequent pattern mining scenarios where each item in one transaction may appear only once, online shopping behaviors tend to repeat themselves. For example, before a user purchases on Taobao, he browses 20 pages on Taobao and 10 pages on JD. Mining frequent patterns with repeated items makes this problem more complicated to analyze.

Leveraging the EFIM (EFficient high-utility Itemset Mining) algorithm [32], an efficient solution to one of the extension problems of frequent pattern mining at linear time with low memory, we are able to discover high-utility itemsets (i.e., group of items) in our mobile shopping transaction data containing utility information. The utility information usually refers to quantities and unit price for each item.

In this case, we consider browsing and purchasing behaviors as items with quantities, and the unit price for each item is the same. That means, it was possible to determine multiple behavior patterns which occurred most often.

Table 4 shows the results. We list the top 12 behavior patterns that users tend to exhibit. From the table, we can see that most users remain on the same platform, which demonstrates a certain amount of loyalty to certain shopping platforms. In addition, some users will browse shopping pages without purchasing anything, especially on JD and Taobao. There are also plenty of users who browse pages across multiple platforms to select the best products. Are Users Loyal to Shopping Platforms? Due to the existence of numerous online shopping platforms, people now have multiple choices and would either choose different shopping platforms due to complex reasons, such as nice price, good service and sales or just stay in one platform. Here we will look from the distribution of how many shopping platforms a user will use to simply answer the question whether users are loyal to shopping platforms first.

From Figure 8, we can see that around 67\% users only visited one shopping platform in about one week, which also shows users' loyalty to shopping platforms to some extent.

To Which Extent Are Users Loyal to Shopping Platforms? From the previous subsection, we know that to some extent users are

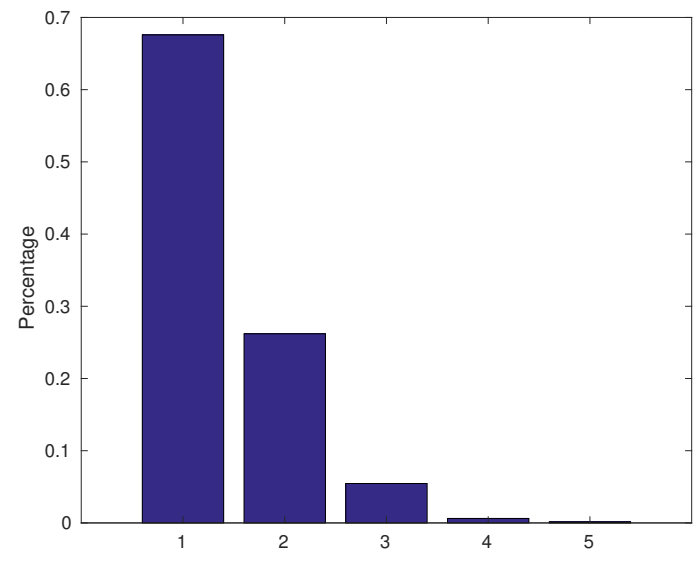

Figure 8: Distribution of users who have cross-platform behaviors. X-axis: The number of platforms a user has visited. Y-axis: Percentage.

loyal to shopping platforms. But the degree of loyalty to these platforms is still unknown. In order to answer this query, we will attempt to build a model to calculate users' loyalty to shopping platforms.

Oliver [21] defines brand loyalty as "a deeply held commitment to re-buy or re-patronize a preferred product/service consistently in the future, thereby causing repetitive same-brand or same brandset purchasing, despite situational influences and marketing efforts having the potential to cause switching behavior." Thus we consider two types of loyalty during the whole process according to this definition. Firstly, a user's browsing loyalty for each purchase. Before each purchase, a user is free to browse pages on any platform. The browsing loyalty refers to whether a user will only browse a single platform for a purchase or whether a user may move across different platforms to search for the best deals. Secondly, a user's purchasing loyalty in all his purchases, that is to say, whether a user would buy products on the same platform, or whether a user will buy goods from different platforms, such as on Taobao the first time and later place his order on JD.

In order to model a user's browsing loyalty, we consider the pages a user visits during a purchase. We denote $p_{i n}$ as the probability that a user is browsing on the platform $i$ for the purchase $n$, whereas $p_{i}$ can be calculated directly from a user's browsing history. With respect to a user's purchasing loyalty, we denote $q_{i}$ as the probability that a user purchases on the platform $i$. As a result, we can define user loyalty $L$ to shopping platforms as follows:

$$
L=\frac{1}{N} \sum_{n=1}^{N} q_{i} p_{i n}
$$

where $n$ means the $n$th purchase, $N$ is the total number of purchases a user has and $i$ is the platform the user makes his purchase on. For example, if a user has two successful purchases, he made the first purchase on Taobao and the second one on JD. For the first purchase, he browsed 10 shopping pages in total, among which he browsed 2 pages on Taobao. For the second purchase, he also browsed 10 pages and 4 pages on JD. So his loyalty is $1 / 2 *(0.5 * 0.2+0.5 * 0.4)=0.15$. 
We consider two extreme cases to validate our model. In the first case, we presume that a user is quite loyal to one shopping platform and that he carries out all his browsing and purchases on the same platform. Thus, his loyalty is 1 . In the second case, we presume that a user carries out 5 successful purchases, once each on the platforms mentioned above. For each purchase he browsed 10 pages, but only 1 page on the platform where he made his purchase. Thus his loyalty is $0.2 *(0.1 * 0.2) * 5=0.02$, which is quite low. This confirms our assumption.

According to the loyalty definition, we calculate all users' loyalty in our data. We find that more than $99 \%$ of users are loyal with loyalty greater than 0.99 .

\section{THE PREDICTABILITY OF CONSUMERS' PURCHASING BEHAVIORS}

Experimental Setup. In this section, we explore whether the consumer's shopping behaviors are predictable. From previous sections, we have known that most of the users are loyal to the platforms they visited. We can see in Fig. 8 that around $67 \%$ users have only visited one platform. In other words, if we assume that one user would choose to use the most frequent visited platform, we can get the prediction performance with accuracy up to at least $67 \%$. Could the consumer behaviors be better predictable?

With characteristic that lead to consumers' purchase learned from previous sections, we build a prediction model to predict which platform a user would like to purchase on at certain time in this section.

Our problem can be formalized in the following way: Given a number of users who have scanned or purchased in shopping platforms such as "Taobao", "Jingdong”, "Dangdan", "Suning”, "Vip", we have all their past browsing and purchasing records, our goal is to predict which platform the user will use next time to make his purchase?

To address this issue within our dataset, we extract 265,619 records for more than 65,000 users from Apr. 20, 2016 to Aug. 14, 2016. After eliminating the records with no purchases or only with one purchase, we get a sample dataset that contains 102,517 records of 12,384 users.

We randomly select half of all users as training and validation set, which uncovers the most suitable experimental parameters shown in Table 5. We then use the rest half of the users as a test dataset. That is to say, there are 6,192 users in the training set, and the left 6,192 users in the testing set for all experiments.

We have utilized Weka and Tensorflow to train and predict using following algorithms: J48 (C4.5), RandomForest, NaiveBayes, SVM and Long Short-Term Memory (LSTM) Network. All experiments are performed on a PC running ubuntu 16.04 with an Intel Core i5 CPU $(2.8 G H z)$ and $8 G B$ memory.

Features. The features used in our model are extracted based on the observations from previous sections. They can be summarized as follows, which can be divided into four types of features.

Temporal feature: We build a temporal feature to show the time correlation. In practise, we split a day into three periods: sleeping hours (1am - 9am), active hours (9am - 5pm) and spare hours (5pm - 1am). Then we consider users' scan and purchase behaviors in each periods as features.
Table 5: Experiment parameter settings

\begin{tabular}{l|l}
\hline Algorithm & Parameter Settings \\
\hline \hline J48 & $\begin{array}{l}\text { confidence factor } \mathrm{C}=0.5 \\
\text { instance leaf } \mathrm{M}=20\end{array}$ \\
\hline RandomForest & $\begin{array}{l}\text { trees }=100 \\
\text { features per tree }=6\end{array}$ \\
\hline NaiveBayes & Default \\
SVM & $\begin{array}{l}\text { cost }=256 \\
\text { gamma }=0.00048\end{array}$ \\
\hline \multirow{2}{*}{ LSTM } & $\begin{array}{l}\text { learning rate }=0.02 \\
\text { \# neurons }=256 \\
\text { batch size }=50 \\
\text { loss function }=\text { Softmax cross entropy } \\
\text { optimizer }=\text { Adam optimizer }\end{array}$ \\
\hline
\end{tabular}

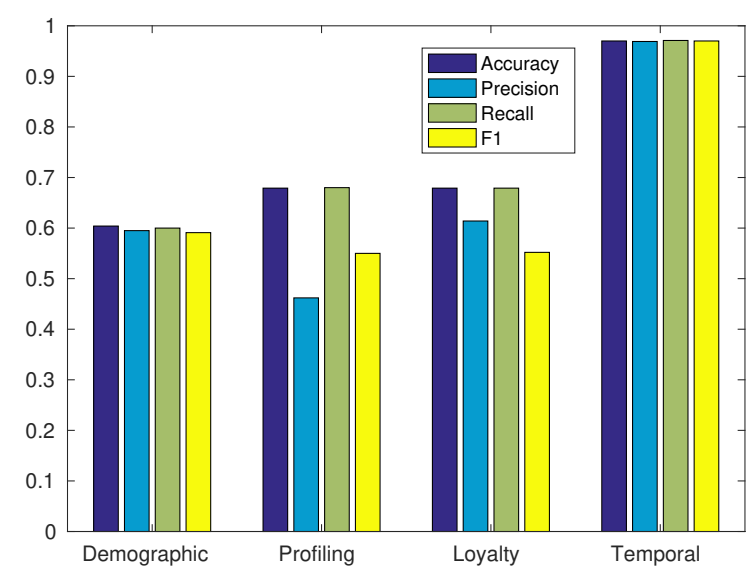

Figure 9: Factor contribution analysis. $\mathrm{X}$-axis represents the four types of features we considered in our prediction framework. Y-axis means the prediction performance.

Loyalty feature: We construct a loyalty feature follows Equ.(1) as described in Section 7.

Profiling feature: We extract the most frequently used apps to profile users' usage behaviors, and use them as profiling features. In practice, users' usage behaviors have been classified into three categories: Human-oriented, Utility-oriented and Entertainment-oriented.

Demographic feature: We consider users' demographic as one feature, such as his location.

Prediction Performance. The prediction results are shown in Table 6.

As we can see, all tested algorithms perform similar performance with F1 score higher than 0.9 , which shows that consumers' crossplatform shopping behaviors are predictable and our prediction performance is sound (far better than random guess).

We examine the contribution of four types of features and check each features' prediction power separately. Figure 9 shows the results. We can see from the figure that temporal feature has the strongest prediction power. 
Table 6: Prediction performance

\begin{tabular}{l|l|l|l|l}
\hline Algorithm & Precision & Recall & F1 & Accuracy \\
\hline J48 & 0.982 & 0.978 & 0.979 & 0.978 \\
\hline RandomForest & 0.980 & 0.980 & 0.980 & 0.979 \\
\hline NaiveBayes & 0.944 & 0.943 & 0.943 & 0.942 \\
\hline SVM & 0.975 & 0.975 & 0.975 & 0.976 \\
\hline LSTM & 0.974 & 0.974 & 0.974 & 0.977 \\
\hline
\end{tabular}

\section{RELATED WORK}

In the past few years mobile phones have witnessed a remarkable evolution and explosive popularization [22]. Meanwhile, ecommerce also has a prosperous development and drastically changed traditional commercial relationships, as well as the shopping process for the fast-growing online shoppers [3]. With a smart phone at hand, the consumer can check the details of products, compare the prices across various e-commerce platforms, save items into charts and enjoy a great many benefits such as personalization from merchants and recommendation from social networks [4, 10, 16, 31]. At the age of information explosion, the complexity of users' online behaviors is increasing while understanding the targets and preferences behind a user's online behavior can provide valuable information for content providers, such as improving user satisfaction by personalizing their contents in search engines and e-commerce web sites [5, 6]. Understanding the consumer buying process can make a difference between success and failure in consumer marketing strategies [26]. On the basis, e-commerce companies can improve their service quality to keep competition ability.

There is plenty of work investigating factors that contribute to successful purchasing results, such as motivation [20] and demographics (e.g. gender, age and residence) [12, 14, 23, 24]. One important research issue is to identify consumers' purchasing intentions from multiple datasets, such as web search contents, social network posts and mobile data cookies. Early researches focused predominantly on identifying web search goals in order to derive commercial information. Dai et al. [6] focused on users' commercial intention from search queries and webpages, i.e., when a user submitted a query or browsed a webpage, whether he/she was about to commit or in the middle of a commercial activity, such as purchase, auction, selling, paid service, etc. Guo et al. [9] proposed an improved method for the detection of a searcher's intention and studied an important practical application - predicting ad clicks for a given search. Guo et al. [10] studied the relationship between social networks and consumer behavior in order to know how individuals' commercial transactions were embedded in their social graphs. Zhang et al. [31] provided an extensive analysis on how users' Facebook profile information correlated to their purchases on eBay, and analyzed the performance of different feature sets and learning algorithms for the task of purchase behavior prediction.

Most previous researches only focused on single e-commerce platform [31]. However, users usually choose to move across various e-commerce platforms to compare the quality, price and service etc. to make their best choices [27], which haven't yet been well explored. The dramatic increase in mobile datasets provide new potential in identifying consumers' purchasing intentions and modelling their behaviors. One related work is [18], where Caroline et al. performed a large-scale cross-platform longitudinal study of user purchase intent and how it evolved. More specifically, the authors tried to understand consumer behaviors leading to successful purchasing across different platforms. However, as the dataset they used was entirely based on Pinterest - a content discovery application which allows users to share their shopping fruits on Pinterest rather than real-world e-commerce platforms, their study may not directly reflect the users' shopping behaviors and therefore reflect biased results, since not everyone is willing to share with others all his shopping behaviors and fruits.

Moreover, users' profiles such as their culture, social and ethical would also influence their behaviors [26]. Researchers pay more attention to users profiling $[2,7,11,13,31]$ and apply them in many areas, such as personalization and recommender systems $[1,15,19$, 25]. In this paper, we will also consider whether users' app usage behaviors would influence their shopping decisions.

To the best of our knowledge, our study is the first one to explore real-world consumer behaviors across diverse e-commerce platforms in depth to identify users' commercial intention and purchasing patterns. We take telecom mobile usage data as our source dataset which comprises comprehensive records of users' shopping platform usage. By analyzing this data, we are able to analyze users' online shopping behaviors across different e-commerce platforms.

\section{CONCLUSIONS}

In this paper, we conducted a comprehensive study on cross-platform mobile shopping behaviors using a real-world, large-scale mobile dataset. We found that most people are loyal to the shopping platforms they visit and they do not move across platforms to select goods they want to buy. In addition, most people are quick purchaser who complete a purchase in less than 30 minutes after first browsing for the item of purchase. Besides, with the patterns learned from this paper, we examine the predictability of users' shopping behaviors on multiple platforms. These findings could provide useful insights for future e-business strategies.

To the best of our knowledge, this is the first work studying cross-platform consumer behavior in depth. Future studies could analyze users' social relationships and how they influence users' shopping decisions.

\section{ACKNOWLEDGMENTS}

This work is supported in part by the National Key Research and Development Program of China under grant No.2016QY02D0302, and National Natural Science Foundation of China under grant No.61433019 and No.U1435217.

\section{REFERENCES}

[1] Gediminas Adomavicius and Alexander Tuzhilin. 1999. User profiling in personalization applications through rule discovery and validation. In Proceedings of the fifth ACM SIGKDD international conference on Knowledge discovery and data mining. ACM, 377-381.

[2] Nilanjan Banerjee, Dipanjan Chakraborty, Anupam Joshi, Sumit Mittal, Angshu Rai, and Balaraman Ravindran. 2012. Towards Analyzing Micro-Blogs for Detection and Classification of Real-Time Intentions.. In ICWSM.

[3] Steven Bellman, Gerald L Lohse, and Eric J Johnson. 1999. Predictors of online buying behavior. Commun. ACM 42, 12 (1999), 32-38.

[4] Mark Brown, Nigel Pope, and Kevin Voges. 2003. Buying or browsing? An exploration of shopping orientations and online purchase intention. European Journal of Marketing 37, 11/12 (2003), 1666-1684. 
[5] Lijing Cheng, Yongquan Fan, Chun Yu, and Yajun Du. 2016. An Improved Trustaware Recommender System for Personalized User Recommendation in Tmall DEStech Transactions on Engineering and Technology Research ICMITE2016 (2016).

[6] Honghua Kathy Dai, Lingzhi Zhao, Zaiqing Nie, Ji-Rong Wen, Lee Wang, and Ying Li. 2006. Detecting online commercial intention (OCI). In Proceedings of the 15 th international conference on World Wide Web. ACM, 829-837.

[7] Xiao Ding, Ting Liu, Junwen Duan, and Jian-Yun Nie. 2015. Mining User Consumption Intention from Social Media Using Domain Adaptive Convolutional Neural Network.. In AAAI. 2389-2395.

[8] Martin Ester, Hans-Peter Kriegel, Jörg Sander, Xiaowei Xu, et al. 1996. A densitybased algorithm for discovering clusters in large spatial databases with noise.. In Kdd, Vol. 96. 226-231.

[9] Qi Guo and Eugene Agichtein. 2010. Ready to buy or just browsing?: detecting web searcher goals from interaction data. In Proceedings of the 33rd international ACM SIGIR conference on Research and development in information retrieval. ACM, 130-137.

[10] Stephen Guo, Mengqiu Wang, and Jure Leskovec. 2011. The role of social networks in online shopping: information passing, price of trust, and consumer choice. In Proceedings of the 12th ACM conference on Electronic commerce. ACM, 157-166.

[11] Vineet Gupta, Devesh Varshney, Harsh Jhamtani, Deepam Kedia, and Shweta Karwa. 2014. Identifying Purchase Intent from Social Posts.. In ICWSM.

[12] Blanca Hernández, Julio Jiménez, and M José Martín. 2011. Age, gender and income: do they really moderate online shopping behaviour? Online Information Review 35, 1 (2011), 113-133.

[13] Bernd Hollerit, Mark Kröll, and Markus Strohmaier. 2013. Towards linking buyers and sellers: detecting commercial intent on twitter. In Proceedings of the 22nd International Conference on World Wide Web. ACM, 629-632.

[14] Dietmar Jannach, Markus Zanker, Alexander Felfernig, and Gerhard Friedrich 2010. Recommender systems: an introduction. Cambridge University Press.

[15] Gabriella Kazai, Iskander Yusof, and Daoud Clarke. 2016. Personalised News and Blog Recommendations based on User Location, Facebook and Twitter User Profiling. In Proceedings of the 39th International ACM SIGIR conference on Research and Development in Information Retrieval. ACM, 1129-1132.

[16] Jure Leskovec, Lada A Adamic, and Bernardo A Huberman. 2007. The dynamics of viral marketing. ACM Transactions on the Web (TWEB) 1, 1 (2007), 5.

[17] Vladimir I Levenshtein. 1966. Binary codes capable of correcting deletions, insertions, and reversals. In Soviet physics doklady, Vol. 10. 707-710.

[18] Caroline Lo, Dan Frankowski, and Jure Leskovec. 2016. Understanding Behaviors that Lead to Purchasing: A Case Study of Pinterest. In Proceedings of the 22nd ACM SIGKDD International Conference on Knowledge Discovery and Data Mining. ACM, 531-540.
[19] Stuart E Middleton, Nigel R Shadbolt, and David C De Roure. 2004. Ontological user profiling in recommender systems. ACM Transactions on Information Systems (TOIS) 22, 1 (2004), 54-88.

[20] Gagandeep Nagra and R Gopal. 2013. An study of Factors Affecting on Online Shopping Behavior of Consumers. International Journal of Scientific and Research Publications 3, 6 (2013), 1-4

[21] Richard L Oliver. 1999. Whence consumer loyalty? the fournal of Marketing (1999), 33-44.

[22] Jacob Poushter. 2016. Smartphone Ownership and Internet Usage Continues to Climb in Emerging Economies. Pew Research Center: Global Attitudes \& Trends (2016).

[23] Paul Resnick and Hal R Varian. 1997. Recommender systems. Commun. ACM 40, 3 (1997), 56-58

[24] Bracha Shapira, Francesco Ricci, Paul B Kantor, and Lior Rokach. 2011. Recommender Systems Handbook. (2011).

[25] Ahu Sieg, Bamshad Mobasher, and Robin Burke. 2007. Web search personalization with ontological user profiles. In Proceedings of the sixteenth ACM conference on Conference on information and knowledge management. ACM, 525-534.

[26] Michael R Solomon. 2014. Consumer behavior: Buying, having, and being. prentice Hall Engelwood Cliffs, NJ.

[27] Efraim Turban, David King, Jae Kyu Lee, Ting-Peng Liang, and Deborrah C Turban. 2015. E-commerce: mechanisms, platforms, and tools. In Electronic Commerce. Springer, 51-99.

[28] Marilyn A Winkleby, Darius E Jatulis, Erica Frank, and Stephen P Fortmann. 1992. Socioeconomic status and health: how education, income, and occupation contribute to risk factors for cardiovascular disease. American journal of public health 82, 6 (1992), 816-820.

[29] Jaewon Yang and Jure Leskovec. 2011. Patterns of temporal variation in online media. In Proceedings of the fourth ACM international conference on Web search and data mining. ACM, 177-186.

[30] Nicholas Jing Yuan, Yu Zheng, Xing Xie, Yingzi Wang, Kai Zheng, and Hui Xiong. 2015. Discovering urban functional zones using latent activity trajectories. IEEE Transactions on Knowledge and Data Engineering 27, 3 (2015), 712-725.

[31] Yongzheng Zhang and Marco Pennacchiotti. 2013. Predicting purchase behaviors from social media. In Proceedings of the 22nd international conference on World Wide Web. ACM, 1521-1532.

[32] Souleymane Zida, Philippe Fournier-Viger, Jerry Chun-Wei Lin, Cheng-Wei Wu, and Vincent S Tseng. 2015. EFIM: a highly efficient algorithm for high-utility itemset mining. In Mexican International Conference on Artificial Intelligence. Springer, $530-546$. 